\title{
Urinary Tract Infection due to Extended-Spectrum Beta-Lactamase Producing Organisms is a Risk Factor for Acute Kidney Injury among Patients with Type 2 Diabetes Mellitus
}

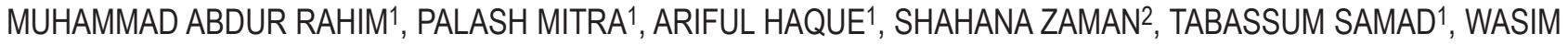 \\ MD. MOHOSINUL HAQUE ${ }^{1}$, MEHERUBA ALAM ANANNA ${ }^{1}$, TUFAYEL AHMED CHOWDHURY' ${ }^{1}$, SHUDHANSHU KUMAR \\ SAHA ${ }^{1}$, SARWAR IQBAL, ${ }^{1}$ SHYAMAL SARKER, ${ }^{3}$ KHWAJA NAJIM UDDIN, ${ }^{4}$ MOHAMMAD ABUL MANSUR ${ }^{1,5}$
}

\begin{abstract}
:
Background: Urinary tract infection (UTI) is common and diabetic patients are at increased risk for UTI. UTI may be complicated by acute kidney injury (AKI). This study was designed to evaluate whether UTI due to extend ed-spectrum beta-lactamase (ESBL) producing organisms should be considered as a risk factor for AKI in type 2 diabetic subjects.

Methods: This case-control study was done in a tertiary care hospital in Dhaka, Bangladesh from April to June 2016. Type 2 diabetic subjects with culture proven UTI were evaluated. Patients with UTI complicated by AKI were cases and those without AKI were taken as controls. ESBL-positivity of the isolated organisms was evaluated as risk factor for $A K I$.

Results: During the study period, a total of 131 (male to female ratio 1:2.6) type 2 diabetic subjects with culture proven UTI were enrolled. Mean age and mean duration of diabetes were $56.1 \pm 13.3$ and $8.7 \pm 5.4$ years respectively. Escherichia coli $(82,62.6 \%)$ was the commonest aetiological agent followed by Klebsiella pneumoniae(14, 10.7\%). Two-thirds (55/82, 67.1\%) of E. coli and two-fifths (6/14, 42.9\%) of Klebsiellae were ESBL-positive. UTI in 64 (48.9\%) patients were due to ESBL-positive organisms. Out of 131 UTI patients, 62 (47.3\%) had AKI; 40 (40/64, 62.5\%) among ESBL-positive and 22 (22/67, 32.8\%) among non-ESBL organisms. There were no significant difference in relation to age $(p=0.71)$, sex $(p=0.26)$, duration of diabetes $(p=0.37)$ and glycated haemoglobin (HbAlc) ( $p=0.69)$ between cases and controls. ESBL-positivity appeared as a significant risk factor for AKI among the study subjects (OR=3.4, 95\% $C I=1.66-6.99, p=0.008)$.

Conclusions: Almost half of the type 2 diabetic subjects with UTI had ESBL-positive organisms as aetiological agents in this study. UTI due to ESBL-positive organisms was a significant risk factor for AKI.
\end{abstract}

Key words: Acute kidney injury, extended-spectrum beta-lactamase, risk factor, type 2 diabetes mellitus, urinary tract infection.

\section{Introduction:}

Urinary tract infection (UTI) is one of the most common bacterial infections in adults. Female patients and patients with diabetes mellitusare at increased risk for UTI. ${ }^{1,2}$ Escherichia coli and Klebsiella pneumoniae are the two most common organisms responsible for UTI throughout

1. Department of Nephrology, Bangladesh Institute of Research and Rehabilitation in Diabetes, Endocrine and Metabolic Disorders (BIRDEM) General Hospital, Dhaka, Bangladesh.

2. Department of Cardiology, National Institute of Cardiovascular Diseases (NICVD), Dhaka, Bangladesh.

3. Professor of Medicine, Dhaka Medical College, Dhaka.

4. Professor of Internal Medicine, BIRDEM, Dhaka.

5. Transplant Unit, Bangladesh Diabetic Somiti (BADAS), Dhaka, Bangladesh.

Corresponding author: Dr. Muhammad Abdur Rahim, Assistant Professor, Department of Nephrology, BIRDEM General Hospital, Dhaka, Bangladesh. email: muradrahim23@yahoo.com. the world. ${ }^{3-6}$ Extended-spectrum beta-lactamase (ESBL) producing strains are an ever increasing problem ${ }^{7-9}$ and diabetic patients with long duration and poor glycaemic control are at increased risk for UTI due to ESBL-positive organisms. ${ }^{5,10}$ UTI may be complicated by acute kidney injury (AKI) and sepsis. ${ }^{3}$ This study was designed to evaluate whether UTI due to ESBL-positive organisms should be considered as a risk factor for AKI in type 2 diabetic subjects.

Methods:

This case-control study was done in the Department of Internal Medicine and Department of Nephrology of Bangladesh Institute of Research and Rehabilitation in Diabetes, Endocrine and Metabolic Disorders (BIRDEM) General Hospital, Dhaka, Bangladesh from April to June 2016. Hospitalized patients with a clinical diagnosis of UTI were initially enrolled for the study purpose. A clean catch technique was applied for urine (preferably early morning midstream specimen) collection. 
JM Vol. 19, No. 1 Urinary Tract Infection due to Extended-Spectrum Beta-Lactamase Producing Organisms is a Risk Factor for Acute Kidney Injury among Patients with Type 2 Diabetes Mellitus

Collected samples were sent to the microbiology laboratory within half an hour, where these samples were inoculated within two hours in Mac Conkey agar and blood agar. Significant culture positive cases (semi-quantitative colony count $>1 \times 10^{5}$ colony forming units $/ \mathrm{ml}$ ) were then included for the study purpose. Catheterized patients, pregnant ladies, patients with inadequate/no growth or growth of candida on urine culture were excluded from the study. Growth of organisms was further evaluated by their colony characters and biochemical tests including triple sugar iron (TSI), motility indole urea (MIU) and Simon citrate. Then the selected samples were sub-cultured for antibiotic sensitivity in Muellar-Hilton (MH) agar by Kibry-Bauer disc susceptibility test. ${ }^{11}$ ESBL-positivity was determined phenotypically by Double Disc Synergy Test. Besides the urine culture, patients were evaluated clinically and by other required laboratory tests. Whether patients developed AKI was noted. AKI was diagnosed as per Kidney Disease: Improving Global Outcomes (KDIGO) Clinical Practice Guideline 2012. ${ }^{12}$ Then, patients with UTI complicated by
AKI were taken as cases and UTI cases without AKI were controls. Finally, ESBL-positive organisms were evaluated as risk factor for AKI among the study participants. Statistical package for social sciences (SPSS) version 20.0 was used to analyze data. A $p$ value of $<0.05$ was taken as significant. Results were presented in tables.

\section{Results:}

During the study period, a total of 131 type 2 diabetic subjects,including 95 females, with culture proven UTI were enrolled. Mean age and mean duration of diabetes of the study participants were $56.07 \pm 13.30$ and $8.70 \pm 5.39$ years respectively. Sixty two (47.3\%) patients with UTI were complicated by AKI (cases). There was no significant difference regarding age, sex, duration and control of diabetes between cases and controls (Table I). E. coli (82, $62.6 \%$ ) was the commonest aetiological agent followed by Klebsiellae (14, 10.7\%). Two-thirds (55/82, 67.1\%) of E. coli and two-fifths $(6 / 14,42.9 \%)$ of Klebsiellae were ESBL-positive (Table II).

Table-I

Base-line characteristics of the study subjects $(\mathrm{N}=131)$

\begin{tabular}{lcccc}
\hline Characteristics & $\begin{array}{c}\text { Overall } \\
(\mathrm{N}=131)\end{array}$ & $\begin{array}{c}\text { Cases (UTI with } \\
\text { AKI) }(\mathrm{n}=62)\end{array}$ & $\begin{array}{c}\text { Controls (UTI } \\
\text { without AKI) }(\mathrm{n}=69)\end{array}$ & p value \\
\hline Mean age (years) & $56.07 \pm 13.30$ & $55.61 \pm 14.13$ & $56.48 \pm 12.59$ & 0.7100 \\
Male:Female & $1: 2.64$ & $20: 42$ & $16: 53$ & 0.2460 \\
Mean duration of DM (years) & $8.70 \pm 5.39$ & $9.15 \pm 5.40$ & $8.30 \pm 5.39$ & 0.3696 \\
Mean HbA1c (\%) & $8.93 \pm 1.96$ & $9.0 \pm 1.78$ & $8.86 \pm 2.13$ & 0.6857 \\
\hline
\end{tabular}

Table-II

Aetiological agents among the study subjects ( $\mathrm{N}=131)$

\begin{tabular}{lccc}
\hline Bacteria & ESBL-positive & Non-ESBL & Total \\
\hline E. coli & $55(42.0)$ & $27(20.6)$ & $82(62.6)$ \\
K. pneumoniae & $6(4.6)$ & $8(6.1)$ & $14(10.7)$ \\
Pseudomonas spp. & --- & $3(2.3)$ & $3(2.3)$ \\
Citrobacter & --- & $3(2.3)$ & $3(2.3)$ \\
Staphylococcus & --- & $7(5.3)$ & $7(5.3)$ \\
*MRSA Staph & --- & $1(0.8)$ & $1(0.8)$ \\
Enterococcus & --- & $10(7.6)$ & $10(7.6)$ \\
Acinetobacter & --- & $6(4.6)$ & $6(4.6)$ \\
Citrobacter & $1(0.8)$ & --- & $1(0.8)$ \\
Streptococcus & --- & $2(1.5)$ & $2(1.5)$ \\
Enterobacter & $2(1.5)$ & --- & $2(1.5)$ \\
Total & $64(48.9)$ & $67(51.1)$ & $131(100)$ \\
\hline
\end{tabular}

*MRSA $=$ Methicillin resistant Staphylococcus aureus
UTI in $64(48.9 \%)$ patients were due to ESBL positive organisms. Out of 131 UTI patients, 62 (47.3\%) had AKI; $40(40 / 64,62.5 \%)$ among ESBL-positive and $22(22 / 67$, $32.8 \%$ ) among non-ESBL organisms. ESBL-positivity appeared as a significant risk factor for AKI among the study subjects (Table III).

\section{Table-III}

Evaluation of UTI with ESBL-positive organisms as risk-factor for AKI among the study subjects $(\mathrm{N}=131)$

\begin{tabular}{lccc}
\hline Bacteria & $\begin{array}{c}\text { ESBL } \\
\text { positive }\end{array}$ & $\begin{array}{c}\text { Non } \\
\text { ESBL }\end{array}$ & Total \\
\hline ESBL-positive (64) & 40 & 24 & 3.4, \\
Non-ESBL (67) & 22 & 45 & $\begin{array}{c}1.66-6.99, \\
0.008\end{array}$ \\
\hline
\end{tabular}




\section{Discussion:}

The definition of AKI has evolved through different stages and KDIGO defined AKI as having one or more of following 3 criteria: absolute rise of serum creatinine value more than $0.3 \mathrm{mg} / \mathrm{dl}$ in a 48-hour period, a rise of serum creatinine to 1.5 times of base-line in one week and decrease in urine production to less than $0.5 \mathrm{ml} / \mathrm{kg} /$ hour for more than 6 hours. ${ }^{12}$ This is the most updated and widely used criteria for AKI and we used this definition in our study to define AKI. We relied mostly on first two criteria based on serum creatinine values.

AKI is common in hospital settings and more common in intensive care units (ICU). ${ }^{13}$ Patients with diabetes are also at increased risk for AKI. ${ }^{14}$ Past history of AKI and CKD are reported risk factors for AKI. ${ }^{14}$ UTI may be complicated by AKI.,15 Older age, diabetes, upper UTI, poor base-line renal functions are reported risk factors for AKI in UTI. ${ }^{15}$

In our study, we evaluated ESBL-positivity of organisms causing UTI as risk factor for AKI. UTI due to ESBL-positive organisms are increasingly been reported in Bangladesh ${ }^{16-18}$ and Rahim MA et a ${ }^{10}$ reported that diabetic females with long duration and poor glycaemic control are risk factors for UTI due to ESBL-positive organisms. Similar observation was reported in other studies. ${ }^{5,19-22}$ Prior hospitalization and repeated UTI and antibiotic intake were risk factors for UTI due to ESBL-positive organisms in another study. ${ }^{23}$ UTI due to ESBL-positive organisms can deteriorate kidney function in kidney transplant recipients as well. ${ }^{24} \mathrm{We}$ found ESBL-positivity as a significant risk factor for AKI in patients with type 2 diabetes mellitus.

This study had some potential limitations. It was a single center study, the study period was only 3 months and the sample size was small. We did not evaluate other risk factors for AKI like base-line kidney function, concomitant treatment with angiotensin blocking agents i.e. angiotensin converting enzyme inhibitors (ACEIs) or angiotensin receptor blockers (ARBs), hydration status of patients as vomiting is a common feature in $\mathrm{UTI}^{4}$ and whether patients were treated with any antibiotic with nephrotoxic potential like aminoglycoisdes. Taking all these confounders in considerations, a larger, multicenter study will give a more reliable answer to the research question tested in this study. Diabetic patients with UTI complicated by AKI merit long-term follow up for their ultimate renal outcomes, ${ }^{25}$ consideration of this fact was also beyond our capacity in this particular study.

\section{Conclusions:}

In conclusion, it can be said that UTI due to ESBL-positive organisms are increasing; almost half of the type 2 diabetic subjects had UTI due to ESBL-positive agents in this study. UTI due to ESBL-positive uropathogens appeared as significant risk factor for AKI among patients with type 2 diabetes. A good glycaemic control and general measures to prevent UTI may become helpful in prevention of UTI due to ESBL-positive organisms and thus may reduce the risk of AKI among type 2 diabetic patients.

Conflict of interest: None.

\section{References:}

1. Guglietta A. Recurrent urinary tract infections in women: risk factors, etiology, pathogenesis and prophylaxis. Future Microbiol 2017;12:239-246.

2. Aswani SM, Chandrashekar UK, ShivashankaraKN, Pruthvi BC. Clinical profile of urinary tract infections in diabetics and non-diabetics. AMJ 2014;7(1):29-34.

3. Chiu CC, Lin TC, Wu RX, Yang YS, Hsiao PJ, Lee Y, et al. Etiologies of community-onset urinary tract infections requiring hospitalization and antimicrobial susceptibilities of causative microorganisms. J Microbiol Immunol Infect 2016;:S1684-1182(16)30143-8.

4. RahmanMR, Rahim MA, Afroze SR, Afroz F, HaqueHF, Mitra P, et al. Pattern of Bacterial Pathogens Causing Urinary Tract Infection and Their Antibiotic Sensitivity: A Tertiary Care Hospital Experience. Birdem Med J 2015;5(1):20-23.

5. Shaikh S, Fatima J, Shakil S, Rizvi SMD, Kamal MA. Risk factors for acquisition of extended spectrum bata lactamase producing Escherichia coli and Klebsiellae pneumoniae in North-Indian hospitals. Saudi J BiolSci 2015;22:37-41.

6. Farajnia S, Alikhani MY, Ghotasloud R, Naghili B, Nakhlband A. Causative agents and antimicrobial susceptibilities of urinary tract infections in the northwest of Iran. International Journal of Infectious Diseases 2009;13:140-144.

7. Spadafino JT, Cohen B, Liu J, Larson E. Temporal trends and risk factors for extended-spectrum-beta-lactamase producing Escherichia coli in adults with catheter-associated urinary tractinfection. Antimicrob Res Infect Cont 2014;3:39. 
JM Vol. 19, No. 1 Urinary Tract Infection due to Extended-Spectrum Beta-Lactamase Producing Organisms is a Risk Factor for Acute Kidney Injury among Patients with Type 2 Diabetes Mellitus

8. Kang C-I, Cha MK, Kim SH, Ko KS, Wi YM, Chung DR, et al. Clinical and Molecular Epidemiology of Community Onset Bacteremia Caused by Extended-Spectrum Beta-Lactamase-Producing Escherichia coli over a 6-Year Period. J Korean Med Sci 2013;28:998-1004.

9. Fennell J, Vellinga A, Hanahoe B, Morris D, Boyle F, Higgins $\mathrm{F}$, et al. Increasing prevalence of ESBL production among Irish clinical Enterobacteriaceae from 2004 to 2008: an observational study. BMC Infect Disease 2012; 12:116-123.

10. Rahim MA, Mitra P, Zaman S, HabibSH, Afroze SR, Samad T, et al. Frequency, Risk Factors and Antibiotic Sensitivity Pattern of Extended-Spectrum Beta Lactamase Producing Escherichia coli and Klebsiella epneumoniae Causing Urinary Tract Infection: Experience from a Tertiary Care Hospital of Bangladesh. BIRDEM Med J 2017;7(2): 155-159.

11. Bauer AW, Kirby WM, Sherris JC, Turck M. Antibiotic susceptibility testing by a standardized single disk method. Am J Clin Pathol 1966;45(4):493-496.

12. Kidney Disease: Improving Global Outcomes (KDIGO) Acute Kidney Injury Work Group. KDIGO Clinical Practice Guideline for Acute Kidney Injury. Kidney inter., Suppl. 2012;2:1-138.

13. Singh TB, Rathore SS, Choudhury TA, Shukla VK, Singh DK, Prakash J. Hospital-acquired acute kidney injury in medical, surgical, and intensive care unit: A comparative study. Indian J Nephrol 2013; 23(1):24-29.

14. Patschan D, Müller GA. Acute Kidney Injury in Diabetes Mellitus. Int J Nephrol 2016: 6232909. http://dx.doi.org/10.1155/2016/62329096232909.

15. Hsiao C, Yang H, Hsiao M, Hung P, Wang MC. Risk Factors for Development of Acute Kidney Injury in Patients with Urinary Tract Infection. PLoS ONE 2015;10(7):e0133835.

16. Asna SMZH, Akter S, Rahman MM, Mohammad N, Hafez MA. Frequency and Sensitivity of Extended Spectrum Beta-Lactamase Positive Organisms in a Secondary and Tertiary Level Hospital Netwark in Dhaka. J Enam Med Coll 2015;5(2):80-87.

17. Iqbal S, Rahim MA, Samad T, Ananna MA, Mitra P, Chowdhury TA. Extended-Spectrum Beta-Lactamase Producing Escherichia coli and Klebsiella pneumonia are Emerging as Major Pathogens Responsible for Urinary Tract Infection. Bangladesh Crit Care J 2015;3(2):49-52.
18. RahmanMR, Rahim MA, Afroze SR, Poddar CS, Uddin KN. Frequency of Extended-Spectrum Beta-Lactamase Producing Organisms Causing Urinary Tract Infection and Their Antibiotic Sensitivity Pattern. J Medicine 2016;18:3-5.

19. Latifpour M, Gholipour A, Damavandi MS. Prevalence of Extended-Spectrum Beta-Lactamase-Producing Klebsiella pneumoniae Isolates in Nosocomial and Community Acquired Urinary Tract Infections. Jundishapur J Microbiol 2016;9(3):e31179.

20. Ozgunes I, Erben N, Kiremitci A, Kartal ED, Durmaz G, Colak H, et al. The prevalence of extended-spectrum beta lactamase-producing Escherichia coli and Klebsiella pneumoniae in clinical isolates and risk factors. Saudi Med J 2006;27(5):608-612.

21. Rubio-Perez I, Martin-Perez E, Garcia DD, Calvo ML-B, Barrera EL. Extended-spectrum beta-lactamase producing bacteria in a tertiary care hospital in Madrid: epidemiology, risk factors and antimicrobial susceptibility patterns. Emerg Health Threats J 2012;5:11589.

22. Osthoff M, Mc Guinness SL, Wagen AZ, Eisen DP. Urinary tract infections due to extended-spectrum beta-lactamase-producing Gram-negative bacteria: identification of risk factors and outcome predictors in an Australian tertiary referral hospital. International Journal of Infectious Diseases 2015;34:79-83.

23. Fernando MMPSC, Luke WANV, Miththinda JKND, Wickramasinghe RDSS, Sebastiampillai BS, Gunathilake MPML. Extended spectrum beta lactamase producing organisms causing urinary tract infections in Sri Lanka and their antibiotic susceptibility pattern - A hospital based cross sectional study. BMC Infectious Diseases 2017; 17:138.

24. Ramadas P, Rajendran PP, Krishnan P, Alex A, Siskind E, Kadiyala A, et al. Extended-Spectrum-Beta-Lactamase Producing Bacteria Related Urinary Tract Infection in Renal Transplant Recipients and Effect on Allograft Function. PLoS ONE 2014;9(3):e91289.

25. Chiu P, Huang C, Liou H, Wu C, Wang S, Chang C. Long-term renal outcomes of episodic urinary tract infection in diabetic patients. Journal of Diabetes and Its Complications 2013;27:41-43. 